\title{
A THIRD-ORDER NUMERICAL SCHEME WITH UPWIND WEIGHTING FOR SOLVING THE SOLUTE TRANSPORT EQUATION
}

\author{
KANGLE HUANG, JIŘÍ ŠIMŮNEK AND M. TH. VAN GENUCHTEN \\ U.S. Salinity Laboratory, USDA, ARS Riverside, CA, U.S.A.
}

\begin{abstract}
SUMMARY
Solute transport in the subsurface is generally described quantitatively with the convection-dispersion transport equation. Accurate numerical solutions of this equation are important to ensure physically realistic predictions of contaminant transport in a variety of applications. An accurate third-order in time numerical approximation of the solute transport equation was derived. The approach leads to corrections for both the dispersion coefficient and the convective velocity when used in numerical solutions of the transport equation. The developed algorithm is an extension of previous work to solute transport conditions involving transient variably saturated fluid flow and non-linear adsorption. The third-order algorithm is shown to yield very accurately solutions near sharp concentration fronts, thereby showing its ability to eliminate numerical dispersion. However, the scheme does suffer from numerical oscillations. The oscillations could be avoided by employing upwind weighting techniques in the numerical scheme. Solutions obtained with the proposed method were free of numerical oscillations and exhibited negligible numerical dispersion. Results for several examples, including those involving highly non-linear sorption and infiltration into initially dry soils, were found to be very accurate when compared to other solutions. (C) 1997 by John Wiley \& Sons, Ltd.
\end{abstract}

KEY WORDS: third-order; numerical method; transport; upwind weighting

\section{INTRODUCTION}

The quality of groundwater depends upon the quality of water infiltrating the vadose zone and the ensuing physical, chemical and biological reactions and transformations involving this water and its dissolved solutes as they move through the unsaturated zone toward the groundwater table. Solute transport in variably saturated soils and groundwater is generally described using convection-dispersion-type transport equations. Much attention has been devoted to the development of accurate numerical solutions of these equations. Especially challenging has been the formulation of numerical solutions that are free of oscillations and artificial dispersion. The most popular solution techniques remain those based upon finite differences and finite elements. ${ }^{1,2}$ Of these two approaches, finite element methods have become the more popular ones because of their relative ease of considering irregular flow domains and associated boundary conditions.

Whether based upon finite differences or finite elements, typical numerical schemes are the implicit and Crank-Nicolson solution algorithms. The implicit scheme gives a first-order, backward-in-time approximation of the time derivative, and as such may introduce considerable numerical dispersion in computed concentration distributions, especially when relatively sharp fronts are to be simulated. By contrast, the time-centred, second-order accurate Crank-Nicolson 
scheme limits or may even eliminate numerical dispersion, but often at the cost of significant phase errors ${ }^{1}$ which can lead to physically unrealistic oscillations in the numerical solutions. Van Genuchten and $\mathrm{Gray}^{3}$ previously developed a dispersion-corrected linear finite element scheme for solving the transport equation. Their scheme is essentially a time-centred, Crank-Nicolsontype approximation, yet provides a third-order approximation of the time derivative through the use of adjusted dispersion coefficients. As compared to the standard Crank-Nicolson scheme, the dispersion-corrected scheme suffers from less numerical oscillations while at the same time yielding a much more precise description of sharp concentration fronts (i.e., producing less numerical dispersion).

A parallel and increasingly popular approach for limiting or avoiding numerical oscillations has been the use of upstream weighting methods. ${ }^{4-13}$ By introducing corrections to the convective velocities and consequently adding an artificial dispersion to increase the stability, upstream weighting ensures that the calculated concentrations remain physically realistic and free of numerical oscillations (i.e. do not overshoot or undershoot the correct solutions), regardless of grid size and time step. ${ }^{10,14}$ Calculated non-physical values (e.g. negative concentrations) sometimes can cause the computations to abort, for example, when non-linear or multicomponent solute reactions are involved. ${ }^{15,16}$ While upstream weighting may be effective in eliminating oscillatory behaviour, the resulting scheme is also known to lead to numerical dispersion for most practical discretizations (mesh sizes).

The objective of this paper is to develop a numerical solution method for the variably saturated solute transport equation which is not only free of numerical oscillations, but also produces no or limited numerical dispersion. The objective is achieved by first extending the dispersion-corrected numerical scheme of van Genuchten and $\mathrm{Gray}^{3}$ to transport under transient variably saturated flow conditions and non-linear adsorption. Subsequently, the derived third-order approximation of the transport equation will be solved using the upwind Petrov/Galerkin finite element method.

\section{FORMULATION}

The one-dimensional transport of miscible components in a variably saturated medium is generally described with the convection-dispersion equation as follows:

$$
\frac{\partial \theta c}{\partial t}+\frac{\partial \rho s}{\partial t}=\frac{\partial}{\partial x}\left(\theta D \frac{\partial c}{\partial x}\right)-\frac{\partial q c}{\partial x}-\mu_{\mathrm{w}} \theta c-\mu_{\mathrm{s}} \rho s+\gamma_{\mathrm{w}} \theta+\gamma_{\mathrm{s}} \rho-S c_{\mathrm{s}}
$$

where $c$ is the solute concentration in solution $\left[\mathrm{ML}^{-3}\right], s$ is the adsorbed concentration $\left[\mathrm{MM}^{-1}\right], \theta$ is the volumetric water content $\left[\mathrm{L}^{3} \mathrm{~L}^{-3}\right], \rho$ is the soil bulk density $\left[\mathrm{ML}^{-3}\right], D$ is the dispersion coefficient $\left[\mathrm{L}^{2} \mathrm{~T}^{-1}\right], q$ is the Darcian fluid flux density $\left[\mathrm{LT}^{-1}\right], \mu_{\mathrm{w}}$ and $\mu_{\mathrm{s}}$ are first-order decay coefficients $\left[\mathrm{T}^{-1}\right]$ for the liquid and solid phases, respectively; $\gamma_{\mathrm{w}}$ and $\gamma_{\mathrm{s}}$ are zero-order rate constants for the liquid $\left[\mathrm{ML}^{-3} \mathrm{~T}^{-1}\right]$ and solid $\left[\mathrm{T}^{-1}\right]$ phases, respectively; $S$ is a sink term in the water flow equation (e.g. to account for root water uptake) $\left[\mathrm{T}^{-1}\right], c_{\mathrm{s}}$ is the solute concentration of the sink term $\left[\mathrm{ML}^{-3}\right], x$ is distance from the soil surface downward [L], and $t$ is time [T]. The Darcian fluid flux density, $q$, in (1) is defined as

$$
q=-K \frac{\partial h}{\partial x}+K
$$

in which $K$ is the hydraulic conductivity $\left[\mathrm{LT}^{-1}\right]$. For transport in the unsaturated zone, $q, K$ and $\theta$ are generally functions of the soil-water pressure head, $h[\mathrm{~L}]$, which is obtained by solving the 
variably saturated flow equation:

$$
\frac{\partial \theta}{\partial t}=\frac{\partial}{\partial x}\left(K \frac{\partial h}{\partial x}-K\right)-S
$$

The influence of sorption on contaminant transport is potentially important since sorption will affect the contaminant transport velocity in the subsurface. For relatively simple systems, the relationship between the adsorbed concentration, $s$, and the solution concentration, $c$, can be described with a sorption isotherm. In this study we assume that the adsorbed and solution concentrations are always in local equilibrium. A common expression for the equilibrium adsorption isotherm is the non-linear Freundlich equation given by

$$
s=k c^{\eta}
$$

in which $k$ and $\eta$ are empirical coefficients. Note that when the exponent $\eta$ equals 1.0 the Freundlich equation is linear, in which case the parameter $k$ reduces to the familiar solid-liquid phase partitioning coefficient, $k_{\mathrm{d}}\left[\mathrm{L}^{3} \mathrm{M}^{-1}\right]$. When the exponent $\eta$ is different from $1 \cdot 0$, the Freundlich isotherm, and hence also the solute transport equation itself, become non-linear. In that case an iterative solution scheme, similar to the one applied to the variably saturated flow equation, must be used to solve the transport equation.

Substitution of (4) into (1) leads to the final form of the solute transport equation considered in this paper:

$$
\frac{\partial \theta R c}{\partial t}=\frac{\partial}{\partial x}\left(\theta D \frac{\partial c}{\partial x}\right)-\frac{\partial q c}{\partial x}-\mu \theta c+\Gamma
$$

where

$$
\begin{aligned}
& \mu=\mu_{\mathrm{w}}+\frac{\rho k c^{\eta-1}}{\theta} \mu_{\mathrm{s}} \\
& R=1+\frac{\rho k c^{\eta-1}}{\theta}
\end{aligned}
$$

and

$$
\Gamma=\gamma_{\mathrm{w}} \theta+\gamma_{\mathrm{s}} \rho-S c_{\mathrm{s}}
$$

Note that the retardation factor $R$ as defined by (7) is different from its conventional definition (e.g. Reference 17) in which the Freundlich exponent $\eta$ appears as an additional multiplier in the last term of (7). Both $\mu$ and $R$ are non-linear coefficients of the concentration $c$, unless $\eta=1$.

\section{THIRD-ORDER APPROXIMATION IN TIME}

The dispersion-corrected numerical solution of (5) is based on the following third-order accurate finite difference equation in time: ${ }^{3}$

$$
\frac{f^{t+\Delta t}-f^{t}}{\Delta t}=\frac{1}{2}\left[\frac{\partial f}{\partial t}-\frac{\Delta t}{6} \frac{\partial^{2} f}{\partial t^{2}}\right]^{t+\Delta t}+\frac{1}{2}\left[\frac{\partial f}{\partial t}+\frac{\Delta t}{6} \frac{\partial^{2} f}{\partial t^{2}}\right]^{t}
$$

where the superscripts $t$ and $t+\Delta t$ indicate evaluation at the old and new time levels in the numerical solution, and where $\Delta t$ represents the time increment used in the calculations. The 
above equation holds for any third-degree polynomial, $f$, in time. Using $(\theta R c)$ for $f$ in equation (9) gives

$$
\frac{(\theta R c)^{t+\Delta t}-(\theta R c)^{t}}{\Delta t}=\frac{1}{2}\left[\frac{\partial(\theta R c)}{\partial t}-\frac{\Delta t}{6} \frac{\partial^{2}(\theta R c)}{\partial t^{2}}\right]^{t+\Delta t}+\frac{1}{2}\left[\frac{\partial(\theta R c)}{\partial t}+\frac{\Delta t}{6} \frac{\partial^{2}(\theta R c)}{\partial t^{2}}\right]^{t}
$$

The left-hand side of this equation will be associated with the first-order time derivative of the governing transport equation (4). An approximation for the second-order derivative in (10) may be obtained using the rule of differentiation

$$
\frac{\partial^{2}(\theta R c)}{\partial t^{2}}=\frac{\partial}{\partial t}\left[\frac{\partial(\theta R c)}{\partial t}\right]
$$

To simplify (11), we assume momentarily that dispersion and other reaction terms in the transport equation can be neglected as high-order effects, i.e.,

$$
\frac{\partial(\theta R c)}{\partial t} \approx-\frac{\partial(q c)}{\partial x}
$$

Incorporating (12) into (11) leads to

$$
\frac{\partial^{2}(\theta R c)}{\partial t^{2}} \approx-\frac{\partial}{\partial t}\left[\frac{\partial(q c)}{\partial x}\right]
$$

Reversing the order of differentiation of the right-hand side of (13) and expanding the resulting time derivative gives

$$
\frac{\partial^{2}(\theta R c)}{\partial t^{2}} \approx-\frac{\partial}{\partial x}\left(q \frac{\partial c}{\partial t}+c \frac{\partial q}{\partial t}\right)
$$

The second term on the right-hand side of (14) may be neglected when the water flow regime is steady-state, or when temporal changes in the flux, $q$, are relatively small. In general, however, the second term of the right-hand side of (14) should be considered, as is done in our study.

Expanding both sides of (12) and incorporating (7) leads to

$$
\theta R \frac{\partial c}{\partial t}+c\left(\frac{\partial \theta}{\partial t}+\rho k \frac{\partial c^{\eta-1}}{\partial t}\right) \approx-\left(q \frac{\partial c}{\partial x}+c \frac{\partial q}{\partial x}\right)
$$

Next, consider the continuity equation for water flow, i.e.,

$$
\frac{\partial \theta}{\partial t}=-\frac{\partial q}{\partial x}-S
$$

in which $S$ is the source/sink term as defined earlier. Substituting (16) into (15) gives

$$
\theta R \frac{\partial c}{\partial t}-c S+c \rho k \frac{\partial c^{\eta-1}}{\partial t} \approx-q \frac{\partial c}{\partial x}
$$

Differentiating the third term of (17), and rearranging the resulting equation, leads to

$$
q \frac{\partial c}{\partial t}=\frac{q}{\theta R \phi}\left(c S-q \frac{\partial c}{\partial x}\right)
$$


where

$$
\phi=1+\frac{\rho k(\eta-1) c^{\eta-1}}{\theta R}
$$

or with (7)

$$
\phi=1+(\eta-1)\left(1-\frac{1}{R}\right)
$$

Incorporating (18) into (14) leads to the following approximation for the second-order time derivative:

$$
\frac{\partial^{2}(\theta R c)}{\partial t^{2}} \approx-\frac{\partial}{\partial x}\left[\frac{q}{\theta R \phi}\left(c S-q \frac{\partial c}{\partial x}\right)+c \frac{\partial q}{\partial t}\right]
$$

Finally, substituting equations (5) and (21) into (10) yields the following third-order finite difference approximation of the transport equation

$$
\begin{aligned}
\frac{(\theta R c)^{t+\Delta t}-(\theta R c)^{t}}{\Delta t}= & \frac{1}{2}\left[\frac{\partial}{\partial x}\left(\theta D^{-} \frac{\partial c}{\partial x}-q^{-} c\right)-\mu \theta c+\Gamma\right]^{t+\Delta t} \\
& +\frac{1}{2}\left[\frac{\partial}{\partial x}\left(\theta D^{+} \frac{\partial c}{\partial x}-q^{+} c\right)-\mu \theta c+\Gamma\right]^{t}
\end{aligned}
$$

where

$$
D^{-}=D-\frac{q^{2} \Delta t}{6 R \theta^{2} \phi}, \quad D^{+}=D+\frac{q^{2} \Delta t}{6 R \theta^{2} \phi}
$$

and

$$
q^{-}=q-\frac{\Delta t q S}{6 R \theta \phi}-\frac{\Delta t \partial q}{6 \partial t}, \quad q^{+}=q+\frac{\Delta t q S}{6 R \theta \phi}+\frac{\Delta t \partial q}{6 \partial t}
$$

One may verify that the dispersion corrections given by (23) reduce to those derived by van Genuchten and $\mathrm{Gray}^{3}$ when $\phi=1$, i.e., for the case of linear sorption $(\eta=1)$ or no adsorption at all $(R=1)$. Equation (24) shows that now also corrections are implemented for the Darcian fluid flux density, $q$. This result is different from, and more accurate than, the approach originally used by van Genuchten ${ }^{18}$ for solute transport under transient variably saturated water flow condition.

The derivation above holds for Freundlich-type adsorption. One may obtain similar higherorder numerical schemes for any arbitrary non-linear isotherm of the general form

$$
s=F(c)
$$

The retardation factor to be used in (22)-(24) becomes then

$$
R=1+\frac{\rho}{\theta} \frac{F(c)}{c}
$$


The corrected equations for $D$ and $q$ remain the same as (23) and (24) above, provided that $\phi$ is now defined as

$$
\phi=1+\frac{\rho}{\theta R}\left[\frac{\partial F}{\partial c}-\frac{F(c)}{c}\right]
$$

The above derivations are also easily extended to multidimensional transport problems described by

$$
\frac{\partial \theta R c}{\partial t}=\nabla \cdot(\theta \underline{\mathbf{D}} \nabla c-\mathbf{q} c)-\mu \theta c+\Gamma
$$

where $\mathbf{q}$ is the water flux density vector, and the dispersion coefficient tensor:

$$
\underline{\mathbf{D}}=\left[\begin{array}{ccc}
D_{x x} & D_{x y} & D_{x z} \\
D_{y x} & D_{y y} & D_{y z} \\
D_{z x} & D_{z y} & D_{z z}
\end{array}\right]
$$

The corresponding three-dimensional variably saturated flow equation is given by

$$
\frac{\partial \theta}{\partial t}=-\nabla \cdot \mathbf{q}-S
$$

The approximation for the second-order time derivative in (10) is now of the form

$$
\frac{\partial^{2}(\theta R c)}{\partial t^{2}}=-\nabla \cdot\left[\frac{\mathbf{q}}{\theta R \phi}(c S-\mathbf{q} \cdot \nabla c)+c \frac{\partial \mathbf{q}}{\partial t}\right]
$$

Substituting (28) and (31) into (10) results in the following finite difference approximation of the three-dimensional solute transport equation:

$$
\begin{aligned}
\frac{(\theta R c)^{t+\Delta t}-(\theta R c)^{t}}{\Delta t}= & \frac{1}{2}\left[\nabla \cdot\left(\theta \underline{\mathbf{D}}^{-} \nabla c-\mathbf{q}^{-} c\right)-\mu \theta c+\Gamma\right]^{t+\Delta t} \\
& +\frac{1}{2}\left[\nabla \cdot\left(\theta \underline{\mathbf{D}}^{+} \nabla c-\mathbf{q}^{+} c\right)-\mu \theta c+\Gamma\right]^{t}
\end{aligned}
$$

where

$$
\underline{\mathbf{D}}^{ \pm}=\left[\begin{array}{ccc}
D_{x x} & D_{x y} & D_{x z} \\
D_{y x} & D_{y y} & D_{y z} \\
D_{z x} & D_{z y} & D_{z z}
\end{array}\right] \pm \frac{\Delta t}{6 \theta^{2} R \phi}\left[\begin{array}{ccc}
q_{x}^{2} & q_{x} q_{y} & q_{x} q_{z} \\
q_{y} q_{x} & q_{y}^{2} & q_{y} q_{z} \\
q_{z} q_{x} & q_{z} q_{y} & q_{z}^{2}
\end{array}\right]
$$

and

$$
\mathbf{q}^{ \pm}=\left\{\begin{array}{c}
q_{x} \\
q_{y} \\
q_{z}
\end{array}\right\}\left(1 \pm \frac{\Delta t}{6 \theta R \phi} S\right) \pm \frac{\Delta t}{6} \frac{\partial}{\partial t}\left\{\begin{array}{c}
q_{x} \\
q_{y} \\
q_{z}
\end{array}\right\}
$$

in which the coefficient $\phi$ is the same as before, i.e., equation (20) for a Freundlich isotherm, or (27) for an arbitrary isotherm $F(c)$. 


\section{UPWIND/PETROV-GALERKIN FORMULATION}

To avoid numerical oscillation, the third-order finite difference approximation of the transport equation (22) is discretized by using the upwind-weighting Galerkin finite element method as follows:

$$
\begin{aligned}
\int_{0}^{l} \frac{(\theta R c)^{t+\Delta t}-(\theta R c)^{t}}{\Delta t} \phi_{i}(x) \mathrm{dx}= & \int_{0}^{l} \frac{1}{2}\left(\left[\frac{\partial}{\partial x}\left(\theta D^{-} \frac{\partial c}{\partial x}\right)-\mu \theta c+\Gamma\right]^{t+\Delta t}\right. \\
& \left.+\frac{1}{2}\left[\frac{\partial}{\partial x}\left(\theta D^{+} \frac{\partial c}{\partial x}\right)-\mu \theta c+\Gamma\right]^{t}\right) \phi_{i}(x) \mathrm{d} x \\
& -\int_{0}^{l}\left[\frac{1}{2}\left(\frac{\partial q^{-} c}{\partial x}\right)^{t+\Delta t}+\frac{1}{2}\left(\frac{\partial q^{+} c}{\partial x}\right)^{t}\right] w_{i}(x) \mathrm{d} x+\Delta t
\end{aligned}
$$

where $l$ is the length of the flow domain and is assumed to be discretized into $(N-1)$ elements with increment $\Delta x_{i}=\left(x_{i}-x_{i-1}\right), \phi_{i}$ are the standard linear basis functions, and $w_{i}$ are asymmetric weighting functions defined by

$$
\begin{aligned}
& w_{1}(x)=\phi_{1}(\xi)-3 \alpha \phi_{1}(\xi) \phi_{2}(\xi) \\
& w_{2}(x)=\phi_{1}(\xi)+3 \alpha \phi_{1}(\xi) \phi_{2}(\xi)
\end{aligned}
$$

in which the subscripts 1 and 2 represent the end nodes of each finite element, and $\xi$ is the local co-ordinate defined in terms of the global co-ordinate, $x$, as

$$
\xi=\frac{2\left(x-x_{1}\right)}{x_{2}-x_{1}}-1, \quad x \in\left[x_{1}, x_{2}\right]
$$

The weighting factor $\alpha$ in (36) is optimally chosen as ${ }^{4}$

$$
\alpha=\operatorname{coth}\left(\frac{P e}{2}\right)-\frac{2}{P e}
$$

where $P e$ is the grid Peclet number. After substituting the usual finite element approximation for $c$ in (35) and performing the integrations, the following final matrix equation is obtained:

$$
\mathbf{A} \mathbf{c}^{t+\Delta t}=\mathbf{B} \mathbf{c}^{t}+\mathbf{F}
$$

where $\mathbf{A}$ and $\mathbf{B}$ are both tridiagonal matrices, and $\mathbf{c}$ and $\mathbf{F}$ are concentration and flux vectors, respectively. The below-diagonal (b), diagonal (d), and above-diagonal (a) entries of $\mathbf{A}$ are given by

$$
\begin{aligned}
b_{i}= & -\frac{1}{4 \Delta x_{i-1}}\left(\theta_{i-1} D_{i-1}^{-}+\theta_{i} D_{i}^{-}\right)-\frac{1}{12}\left(2 q_{i-1}^{-}+q_{i}^{-}\right)-\frac{\alpha}{4} q_{i-1}^{-}, \quad i=2, N \\
d_{i}= & \frac{1}{4 \Delta x_{i-1}}\left(\theta_{i-1} D_{i-1}^{-}+\theta_{i} D_{i}^{-}\right)+\frac{1}{4 \Delta x_{i}}\left(\theta_{i} D_{i}^{-}+\theta_{i+1} D_{i+1}^{-}\right) \\
& +\frac{\Delta x_{i-1}}{6 \Delta t}\left(\theta_{i-1} U_{i-1}+2 \theta_{i} U_{i}\right)+\frac{\Delta x_{i}}{6 \Delta t}\left(2 \theta_{i} U_{i}+\theta_{i+1} U_{i+1}\right) \\
& +\frac{1}{12}\left(q_{i+1}^{-}-q_{i-1}^{-}\right)+\frac{\alpha}{2} q_{i}^{-}
\end{aligned}
$$




$$
a_{i}=-\frac{1}{4 \Delta x_{i}}\left(\theta_{i} D_{i}^{-}+\theta_{i+1} D_{i+1}^{-}\right)+\frac{1}{12}\left(q_{i}^{-}+2 q_{i+1}^{-}\right)-\frac{\alpha}{4} q_{i+1}^{-}, \quad i=1, N-1
$$

where

$$
U_{i}=R_{i}+\frac{\mu_{i} \Delta t}{2}
$$

The entries of matrix $\mathbf{B}$ are the same as those of $\mathbf{A}$ except that $D^{-}$is replaced by $-D^{+}, q^{-}$by $-q^{+} \alpha$ by $-\alpha$, and $\mu$ by $-\mu$. Finally, the entries of vector $\mathbf{F}$ are given by

$$
\begin{gathered}
f_{1}=\frac{\Delta x_{1}}{6}\left(2 \Gamma_{1}+\Gamma_{2}\right)+J_{0} \\
f_{i}=\frac{1}{6}\left[\Delta x_{i-1}\left(2 \Gamma_{i}+\Gamma_{i-1}\right)+\Delta x_{i}\left(2 \Gamma_{i}+\Gamma_{i+1}\right)\right], \quad 2 \leqslant i \leqslant N-1 \\
f_{N}=\frac{\Delta x_{N-1}}{6}\left(2 \Gamma_{N}+\Gamma_{N-1}\right)-J_{l}
\end{gathered}
$$

in which $J_{0}$ and $J_{l}$ are solute fluxes across the inlet $(x=0)$ and outlet $(x=l)$ boundaries, respectively. The resulting system of equations is solved using the Thomas' algorithm, yielding at each time step the desired concentration vector $\mathbf{c}^{t+\Delta t}$.

\section{NUMERICAL TESTS}

This section presents results of several numerical tests to illustrate the performance of the proposed numerical algorithm and its relative accuracy. The algorithm was implemented in the computer code HYDRUS (version 5.0) ${ }^{19}$ which is a revised and updated version of the original HYDRUS code developed by Kool and van Genuchten. ${ }^{20}$ The updated code simulates onedimensional water flow, solute transport and heat movement in a variably saturated porous medium using linear finite elements.

All numerical examples were run assuming a solute flux boundary condition at the soil surface $(x=0)$ and a zero concentration gradient condition at the bottom boundary $(x=l)$. For simplicity, each simulation assumed a uniform initial concentration profile. The proposed numerical algorithm was first verified for an idealized solute transport scenario for which an analytical solution is available. The algorithm was then applied to transport problems involving transient variably saturated water flow and non-linear adsorption. Solutions obtained with the proposed method will be compared with the commonly used first-order implicit schemes. The solution domain in all cases was assumed to be $200 \mathrm{~cm}$ in length and, except where indicated, discretized using uniform spatial increments, $\Delta x$, of $2 \mathrm{~cm}$. Molecular diffusion was assumed to be negligible during the calculation, giving the following approximation for the dispersion coefficient:

$$
D=\varepsilon \frac{|q|}{\theta}
$$

where $\varepsilon$ is the dispersivity $(\mathrm{L})$ of the medium. 
Steady-state flow

The first test problem deals with solute transport in a medium having a constant convective velocity $(v=q / \theta)$ of $4 \mathrm{~cm} / \mathrm{d}$, and a relatively low dispersivity $(\varepsilon)$ of $0.04 \mathrm{~cm}$. No solute sorption, i.e., $s=0$, was considered in this example. A tracer solution having a relative concentration, $C_{0}$, of 1. 0 was applied to an initially solute-free medium for a period of $20 \mathrm{~d}$, after which solute-free water was again added. The grid Peclet number, defined as $P e=v \Delta x / D=\Delta x / \varepsilon$, was equal to 25, which represents a case of convection-dominated transport. A constant time step $\Delta t=0 \cdot 1$ day was used.

Figure 1 shows that the solutions obtained with the proposed method (Central-UW) using $\Delta x=1 \mathrm{~cm}$ is completely free of numerical oscillations. The implicit methods, with and without upwind weighting, also produced stable solutions. The implicit algorithm without upwind weighting suffered from the least numerical dispersion for this example, while the proposed method caused less numerical dispersion than the upwind weighted implicit scheme. Notice that the dispersion-corrected algorithm without upwind weighting predicted the concentration fronts accurately, albeit with some serious oscillations. When the Peclet number was increased to $100(\Delta x=2 \mathrm{~cm}$ and $\varepsilon=0.02 \mathrm{~cm})$, the implicit method without upwind weighting became unstable (Figure 2), while the proposed method still produced stable solutions (see Figure 2).
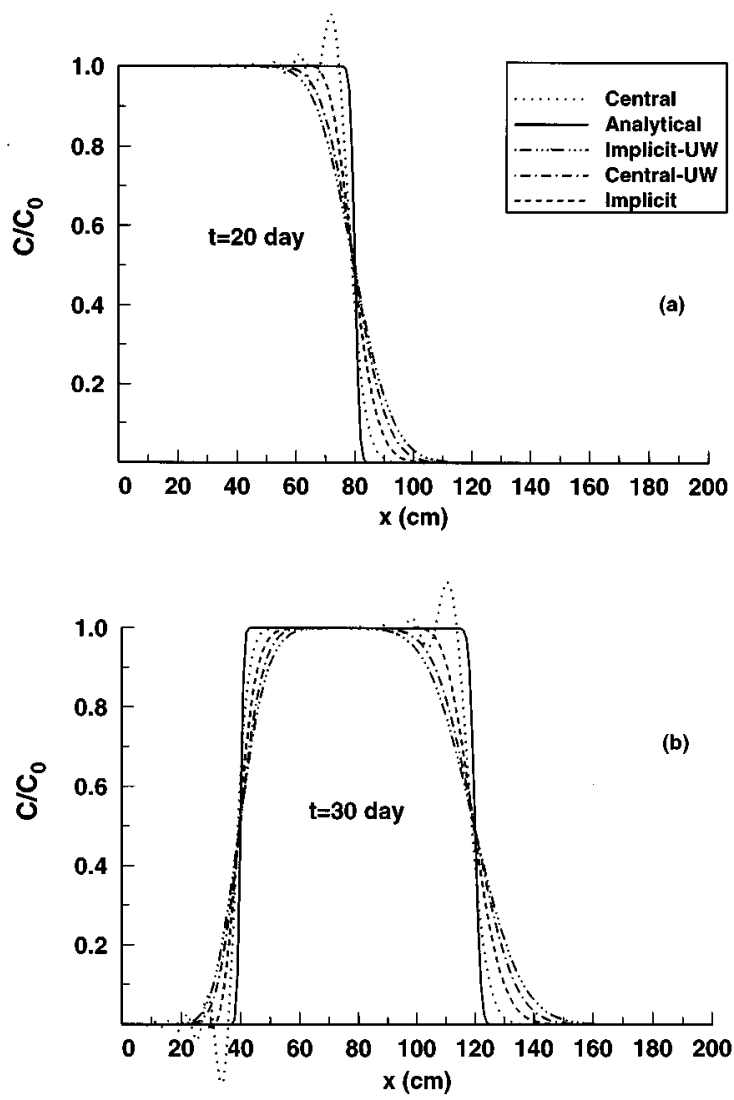

Figure 1. Concentration distributions calculated with the proposed method (Central-UW), the third-order algorithm (Central), the implicit algorithm (Implicit), the upwind weighted algorithm (Implicit-UW), and the exact solution (steadystate flow; $P e=25)$ 

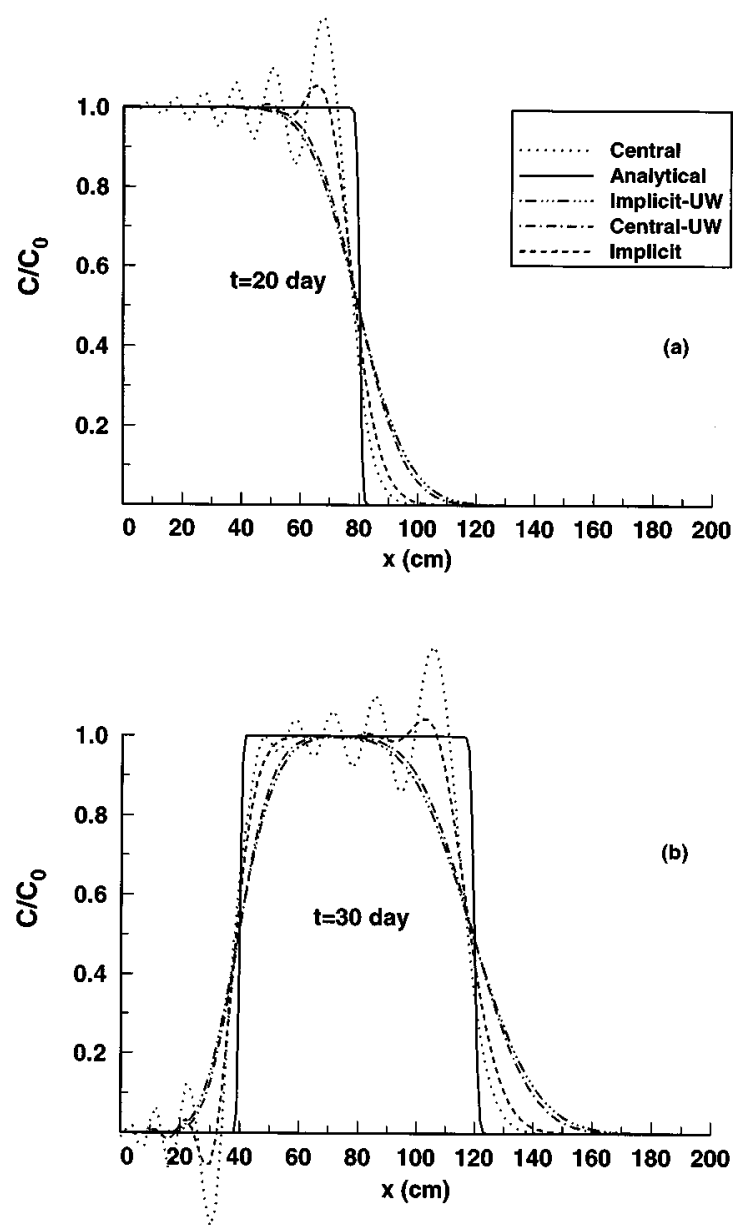

Figure 2. Comparison of calculated concentration distributions obtained with the proposed method and several other numerical algorithms (steady-state flow; $P e=100$ )

Moreover, the numerical dispersion induced by the proposed scheme is again somewhat smaller than that generated with the upwind-weighted implicit algorithm.

\section{Steady-state flow and nonlinear adsorption}

The second example deals with the transport of a solute subject to non-linear adsorption. We used for this example the Freundlich isotherm (4) with coefficient values $k=0.5$ and $\eta=1 \cdot 5$, thus representing a rather strongly non-linear case. The flow and transport conditions were exactly the same as above example. Because of the presence of a non-linear isotherm, the scheme required numerical iterations. Convergence was assumed to be reached when the absolute error in calculated concentrations between two consecutive iterations became less than 0.0001. Varying time steps were employed in this and the following examples. The simulation started by using an initial time step, $\Delta t_{0}=10^{-5} \mathrm{~d}$. The $i$ th time step $\Delta t_{i} \in\left[\Delta t_{\min }, \Delta t_{\max }\right]$ was automatically set according to the iteration convergency history at the previous time step, that is, if the iteration for 

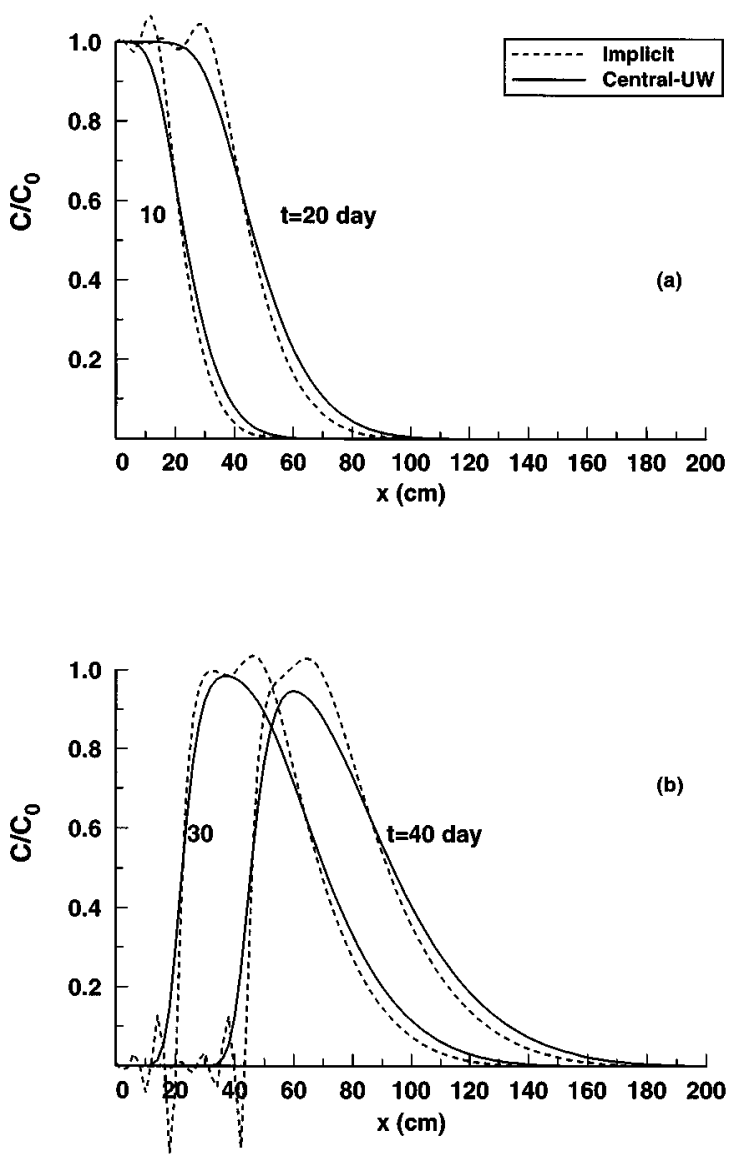

Figure 3. Concentration distributions obtained with the proposed method and the implicit scheme (steady-state flow and non-linear adsorption; $P e=20$ ).

the previous time step converged slowly, the current time step is reduced to be smaller than the previous one; otherwise the current time step is stretched to be larger than the previous. The minimum time step $\Delta t_{\min }=10^{-6} \mathrm{~d}$ and the maximum one $\Delta t_{\max }=0 \cdot 1$ day were arbitrarily chosen in our tests. Shown in Figure 3 are calculated concentration distributions for $P e=20$ $(\Delta x=2 \mathrm{~cm}$ and $\varepsilon=0 \cdot 1 \mathrm{~cm})$. Numerical solutions obtained with the proposed (Central-UW) scheme were completely free of numerical oscillations, even near the concentration peaks which generally are the most vulnerable to non-physical oscillations. By contrast, solutions obtained with the widely-used backward implicit finite element scheme suffered from serious numerical oscillations. Considerable overshoot and especially undershoot were produced with the implicit algorithm for $P e=100(\varepsilon=0.02 \mathrm{~cm})$, while the proposed method still worked very well (Figure 4).

\section{Transient flow and non-linear adsorption}

The above comparisons pertain to transport during steady-state water flow. Simulations were also carried out for solute transport under highly transient flow conditions. We considered 

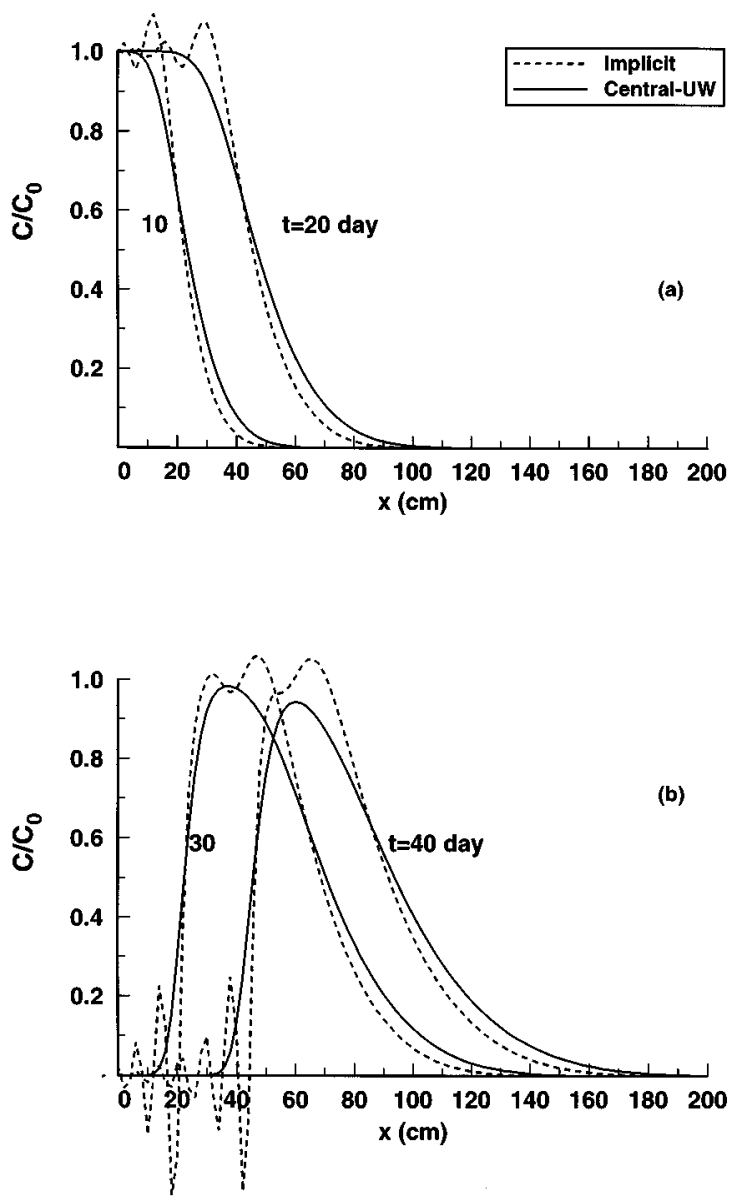

Figure 4. Simulated concentration distributions obtained with the proposed method (Central-UW) and the implicit algorithm (steady-state flow and non-linear adsorption; $P e=100$ )

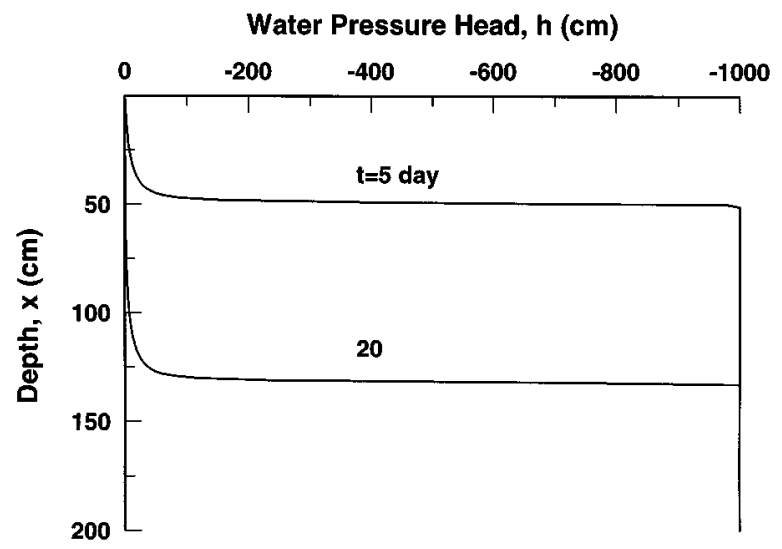

Figure 5. Calculated pressure head distributions for ponded infiltration in a loamy soil 
ponded infiltration into an initially relatively dry $\left(-10^{3} \mathrm{~cm}\right.$ of water pressure head) loamy soil. The soil-hydraulic properties were described with the closed-form functions of van Genuchten $^{21}$ assuming hydraulic parameters $\alpha=0.015(1 / \mathrm{cm}), n=2 \cdot 0, \theta_{\mathrm{s}}=0.45, \theta_{\mathrm{r}}=0.05$, and $K_{\mathrm{s}}=2 \mathrm{~cm} / \mathrm{d}$. An initially square concentration distribution (Figure $6 \mathrm{a}, t=0$ ) was assumed in this example. The solute was again subjected to Freundlich-type non-linear equilibrium adsorption $(k=0.5$ and $\eta=1.5)$. The assumed transport experiment started by applying solute-free irrigation water (ponding) to the soil surface for a period of 20 days. This leaching process was followed by adding water having a solute concentration of 1.0 to the soil profile. Correct simulation of this example by means of standard numerical methods can be very difficult because of the highly non-linear nature of both the flow and transport process. For each time step, accurate solutions for the pressure head, $h$, and the water content, $\theta$, were first obtained by solving the mixed-form Richards equation using the modified Picard iteration algorithm ${ }^{22}$ coupled with a newly formulated convergence criterion. ${ }^{23}$ Procedures of numerical iteration for the non-linear transport solution are the same as the previous example. Figure 5 shows calculated pressure head distributions during the leaching process. Notice the very sharp distributions of the moisture front. Calculated flux density $q$ by Darcy's law (2) from the $h$-profiles in Figure 5 were subsequently used in numerical transport algorithm to obtain solutions of solute concentration.
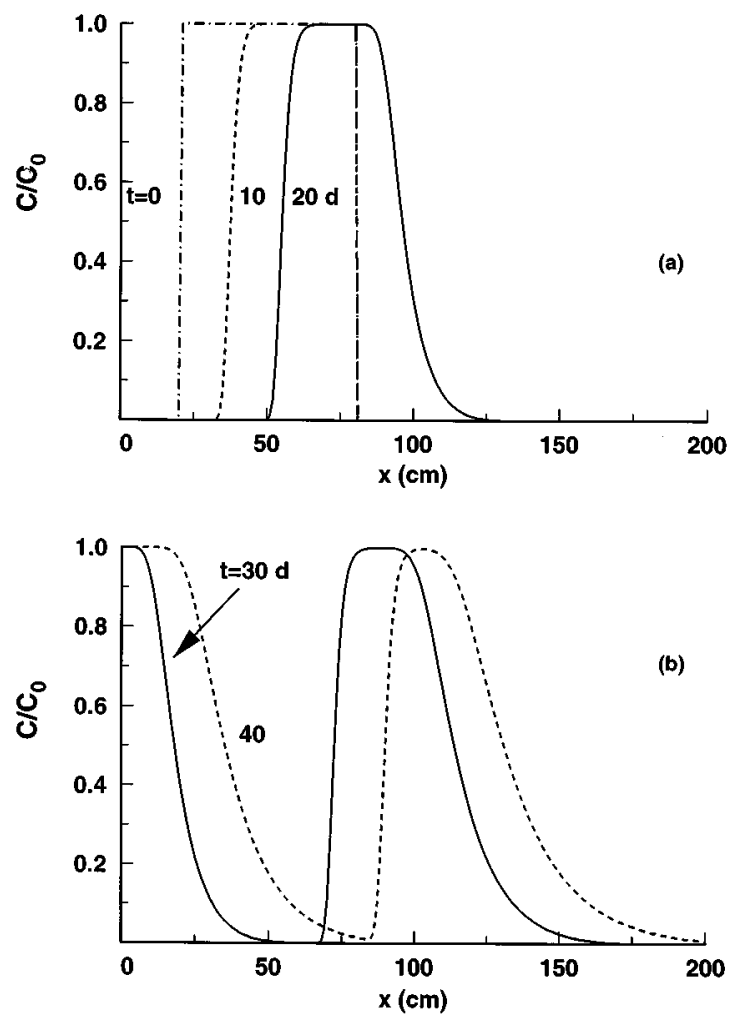

Figure 6. Calculated concentration distributions obtained with the proposed method (Central-UW) for solute transport during transient infiltration and involving non-linear adsorption $(P e=20)$ 


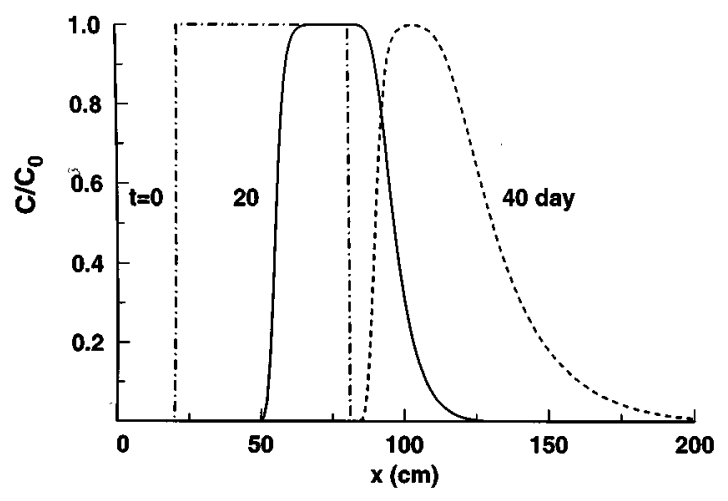

Figure 7. Calculated concentration profiles obtained with the proposed method for transport during transient flow and involving nonlinear adsorption $(P e=100)$

Figure 6 presents predicted concentration distributions for $P e=20(\Delta x=1 \mathrm{~cm}, \varepsilon=0 \cdot 05 \mathrm{~cm})$. The results show that the proposed Central-UW method produced very stable solutions, i.e., being oscillation-free and having very little if any numerical dispersion. Notice that the concentration fronts are very steep, which is typical for cases where the numerical solutions are most vulnerable to both numerical oscillations and artificial dispersion. This example was purposely designed so as to test the performance of the proposed method in situations where multiple solute peaks are present. A relatively poor performance of a numerical scheme in such situations often leads to peak clipping (yielding lower concentration peaks) and valley elevation (producing relatively higher minimum concentrations) as compared to the correct solutions. ${ }^{24}$ Figure 7 shows that numerical solutions for $P e=100(\varepsilon=0.01 \mathrm{~cm})$ also produced excellent results. Finally, we note that for all of the simulations discussed above we obtained extremely good mass conservation, with mass balance errors always being less than $0 \cdot 1$ per cent. Although a good mass balance per se does not necessarily mean that the predicted solutions are accurate, a correct mass balance is at least a requisite for an accurate numerical solution.

\section{CONCLUSIONS}

An accurate third-order approximation of the solute transport equation was derived. The scheme leads to corrections for both the dispersion coefficient and the convective velocity in the solute transport equation. The algorithm is an extension of previous work by van Genuchten and Gray ${ }^{3}$ and van Genuchten ${ }^{18}$ to conditions involving transient fluid flow and non-linear adsorption. The third-order algorithm accurately describes concentration distributions near sharp concentration fronts, thereby showing its ability to eliminate artificial dispersion. Numerical oscillations in the algorithm could be avoided by employing upwind weighting. Solutions obtained with the proposed method were completely free of numerical oscillations and exhibited negligible numerical dispersion. Results for several examples involving non-linear sorption and infiltration into a very dry soil, were found to be very accurate when compared to other solution methods.

\section{REFERENCES}

1. G. F. Pinder and W. G. Gray, Finite Element Simulation in Surface and Subsurface Hydrology, Academic Press, New York, 1977.

2. L. Lapidus and G. F. Pinder, Numerical Solution of Partial Differential Equations in Science and Engineering, John Wiley \& Sons, New York, 1982. 
3. M. Th. van Genuchten and W. G. Gray, 'Analysis of some dispersion corrected numerical schemes for solution of the transport equation', Int. j. numer. methods eng., 12, 387-404 (1978).

4. J. C. Heinrich, P. S. Huyakorn, O. C. Zienkiewicz and A. R. Mitchell, 'An 'upwind' finite element scheme for two-dimensional convective-transport equations', Int. j. numer. methods eng., 11, 131-143 (1977).

5. B. P. Leonard, 'A survey of finite differences of opinion on numerical modeling of the incomprehensible defective confusion equation', Finite Element Methods for Convection Dominated Flows, Annual Meeting of the ASCE, New York, 1979, pp. 1-17.

6. P. S. Huyakorn and K. Nilkuha, 'Solution of transient transport equation using an upstream finite element scheme', Appl. Math. Modelling, 1, 187-195 (1979).

7. A. N. Brooks and T. J. R. Hughes, 'Streamline upwind/Petrov-Galerkin formulations for convection dominated flow with particular emphasis on the impressible Navier-Stokes equations', Comput. Methods Appl. Mech. Eng., 32, 199-259 (1982).

8. N.-Z. Sun and W. W.-G. Yeh, 'A proposed upstream weight numerical method for simulating pollutant transport in groundwater', Water Resource Res., 19, 1489-1500 (1983).

9. A. Mizukami and T. J. R. Hughes, 'A Petrov-Galerkin finite element method for convection dominated flows: an accurate upwinding technique for satisfying the maximum principle', Comput. Methods Appl. Mech. Eng., 50 (1985).

10. P. H. Sammon, 'An analysis of upstream weighting', Soc. Petrol. Eng. J. Res. Eng., 3, 1053-1056 (1988).

11. J. J. Westerink and D. Shea, 'Consistent higher degree Petrov-Galerkin methods for the solution of the transient convection-diffusion equation', Int. j. numer. methods eng., 28, 1077-1101 (1989).

12. M. Putti, W. W.-G. Yeh and W. A. Mulder, 'A triangular finite volume approach with high-resolution upwind terms for the solution of groundwater transport equations', Water Resource Res., 26, 2865-2880 (1990).

13. C. T. Miller and F. H. Cornew, 'A Petrov-Galerkin method for resolving advection-dominated transport', in T. F. Russell, R. E. Ewing, C. A. Brebbia, W. G. Gray, G. F. Pinder (eds.), Numerical Methods in Water Resources, Vol. 1, Proc. Computational Methods in Water Resources IX, 1992, pp. 157-164.

14. F. W. Letniowski and P. A. Forsyth, 'A control volume finite element method for three-dimensional NAPL groundwater contamination', Int. j. numer. methods fluids, 13, 955-970 (1991).

15. P. A. Forsyth, 'Three-dimensional modeling of steam flush for DNAPL site remediation', Int. j. numer. methods fluids, 19, 1055-1081 (1994).

16. J. Simunek and D. L. Suarez, 'Modeling of carbon dioxide transport and production in soil, 1. Model development', Water Resource Res., 29, 487-497 (1993).

17. M. Th. van Genuchten and R. W. Cleary, 'Movement of solutes in soil: Computer-simulated and laboratory results', in G. H. Bolt (ed.), Soil Chemistry, B. Physico-Chemical Models, Elsevier, Amsterdam, 1979, pp. 349-386.

18. M. Th. van Genuchten, 'Mass transport in saturated-unsaturated media: one-dimensional solutions', Research Report 78-WR-11, Water Resources Program Princeton University, 1978

19. T. Vogel, K. Huang, R. Zhang and M. Th. van Genuchten, 'The HYDRUS (version 5.0) code for simulating one-dimensional water flow, solute transport, and heat movement in variably-saturated media', Research Report No. 140, U.S. Salinity Laboratory, USDA, ARS, Riverside, California, 1996.

20. J. B. Kool and M. Th. van Genuchten, 'HYDRUS (Version 3.3): One-dimensional variably saturated flow and transport model, including hysteresis and root water uptake', Research Report No. 124, U.S. Salinity Laboratory, USDA, ARS, Riverside, CA, 1991.

21. M. Th. van Genuchten, 'A closed-form equation for predicting the hydraulic conductivity of unsaturated soils', Soil Sci. Soc. Am. J., 44, 892-898 (1980).

22. M. A. Celia, E. T. Bouloutas and R. L. Zarba, 'A general mass-conservative numerical solution for the unsaturated flow equation', Water Resource Res., 26, 1483-1496 (1990).

23. K. Huang, B. P. Mohanty and M. Th. van Genuchten, 'A new convergence criterion for the modified Picard iteration method to solve the variably-saturated flow equation', J. Hydrol, 178, 69-96 (1996).

24. G. T. Yeh, J. R. Chang, H. P. Cheng and C. H. Sung, 'An adaptive local grid refinement based on the exact peak capture and oscillation free scheme to solve transport equations', Comput. Fluids, 24, 293-332 (1995). 